\title{
Dynamical Symmetry and Quantum Information Processing with Electromagnetically Induced Transparency
}

\author{
Xiong-Jun Liu ${ }^{a, b 1}$, Hui Jing ${ }^{c}, \mathrm{Xin} \mathrm{Liu}^{a, b}$ and Mo-Lin Ge ${ }^{a, b}$ \\ a. Theoretical Physics Division, Nankai Institute of Mathematics,Nankai University, Tianjin 300071, \\ P.R.China \\ b. Liuhui Center for Applied Mathematics, Nankai University and Tianjin University, Tianjin \\ 300071, P.R.China \\ c. State Key Laboratory of Magnetic Resonance and Atomic and Molecular Physics, \\ Wuhan Institute of Physics and Mathematics, CAS, Wuhan 430071, P. R. China
}

\begin{abstract}
We study in detail the interesting dynamical symmetry and its applications in general manylevel and many-ensemble atomic systems with electromagnetically induced transparency (EIT). By discovering the symmetrical Lie group of various atomic systems, the novel applications to quantum memory and quantum entanglement between photons or atomic ensembles are investigated.

PACS numbers: 03.67.-a, 03.65.Fd, 03.67.Mn, 42.50.Gy
\end{abstract}

\section{Introduction}

During the last decade or so, rapid advances have been witnessed in both experimental and theoretical aspects towards probing the novel mechanism of Electromagnetically Induced Transparency (EIT) [1] and its many potential applications 2, 3, 4, In particular, based on the elegant "dark-state polaritons" (DSPs) theory proposed by Fleischhauer and Lukin [5], the quantum memory techniques are now actively explored by exchanging the quantum state information between the quantized light field and the metastable collective atomic field [6. DSP is a new quantum field which is the superposition of the light field amplitude and the atom coherence between two lower levels of the $\Lambda$ type three-level atoms, and it describes the total system of the optical and collective atomic fields. In linear theory where the two-photon detuning of the light pulses is zero, the dynamical evolution of DSPs can lead to a perfect state mapping from the photonic branch into the atomic excitation one and vice versa by adiabatically adjusting the coupling laser [5, 6].

The dynamical symmetry of multi-level atomic system interacting with light fields was studied by D. A. Lidar et al. 7]. On other hand, a semidirect product group in three-level atomic system under the condition of larger atom number and low collective excitation limit [5] with EIT was discovered by Sun et al [8], and the the validity of adiabatic passage condition for the dark states is also investigated in this technique. After that, a series research on the study of hidden symmetry as well as its application to quantum information with four-level atomic system and many atomic ensembles were done recently 9. 10]. All these works indicate many interesting hidden symmetrical properties in various atomic systems with EIT. In this paper, by discovering the symmetrical Lie group, we examine in detail the general definition of dark-state polariton (DSP) operators, and then the dark-states in different atomic systems. Also, it is interesting to find that the symmetrical properties of the multi-level system and multi-atomic-ensemble system are dependent on some characteristic parameters such as the coupling constant $g_{i}$ and Rabi frequency $\Omega_{i}$ etc.. Furthermore, a controllable scheme to generate

\footnotetext{
${ }^{1}$ Electronic address:xiongjunliu@yahoo.com.cn
} 
quantum entanglement between atoms or lights via quantized DSPs theory is discussed, which might be experimentally implemented in the near future..

The development herein is outlined as follows. In section II, we discuss the dynamical symmetry by discovering the Lie algebra structure of various atomic systems including multi-level and multiatomic ensembles cases etc.; In section III, we respectively examine the general definition of DSP operators and then quantum memory for photons via DSP theory of these systems; Generation of different formalisms of entanglement between atoms or lights via quantized DSPs theory are discussed in section IV; In the last section, we conclude and further discuss the dynamical symmetry and the applications in these EIT-systems.

\section{Hidden symmetrical group in electromagnetically induced transparency}

\subsection{Complex $m$-level $(m>3$, multi-level) atomic system}

The system we consider is shown in Fig. 1 (a), a collection of $N$ double $\Lambda$ type $m$-level ( $m \geq 3$, multilevel) atoms interact with $m-2$ single-mode quantized fields which couple the transitions from the ground state $|b\rangle$ to excited state $\left|e_{\sigma}\right\rangle(1 \leq \sigma \leq m-2)$ with coupling constants $g_{\sigma}$, and $m-2$ classical control ones, which couple the transitions from the metastable state $|c\rangle$ to excited one $\left|e_{\sigma}\right\rangle$ with timedependent Rabi-frequencies $\Omega_{\sigma}(t)$. Generalization to multi-mode probe pulse case is straightforward. Considering all transitions at resonance, the interaction Hamiltonian of the total system can be written as:

$$
\hat{H}=\sum_{\sigma=1}^{m-2} g_{\sigma} \sqrt{N} \hat{a}_{\sigma} \hat{E}_{\sigma}^{\dagger}+\sum_{\sigma=1}^{m-2} \Omega_{\sigma} \hat{T}_{e_{\sigma} c}+h . c .,
$$

where subscription $\sigma$ denotes the corresponding excited state and the collective atomic excitation operators:

$$
\hat{E}_{\sigma}=\frac{1}{\sqrt{N}} \sum_{j=1}^{N} \hat{\sigma}_{b e_{\sigma}}^{j}, \hat{C}=\frac{1}{\sqrt{N}} \sum_{j=1}^{N} \hat{\sigma}_{b c}^{j},
$$

with $\hat{\sigma}_{\mu \nu}^{j}=|\mu\rangle_{j j}\langle\nu|\left(\mu, \nu=b, c, e_{1}, e_{2}, \ldots, e_{m-2}\right)$ being the flip operators of the $j$-th atom between states $|\mu\rangle$ and $|\nu\rangle$, and

$$
\hat{T}_{\mu \nu}^{-}=\hat{T}_{\mu \nu}=\sum_{j=1}^{N} \hat{\sigma}_{\mu \nu}^{j}, \quad \hat{T}_{\mu \nu}^{+}=\left(\hat{T}_{\mu \nu}^{-}\right)^{\dagger},
$$

where $\mu \neq \nu=c, e_{1}, e_{2}, \ldots, e_{m-2}$. Denoting by [1] $|b\rangle=\left|b_{1}, b_{2}, \ldots, b_{N}\right\rangle$ the collective ground state with all $N$ atoms staying in the same single particle ground state $|b\rangle$, we can easily give other quasi-spin wave states by the operators defined in formula (2): $\left|e_{\sigma}^{n}\right\rangle=[n !]^{-1 / 2}\left(\hat{E}_{\sigma}^{\dagger}\right)^{n}|b\rangle$ and $\left|c^{n}\right\rangle=[n !]^{-1 / 2}\left(\hat{C}^{\dagger}\right)^{n}|b\rangle$. For the EIT case, we consider two approximation conditions [5] 6]: i) The system include a very large number of atoms, i.e. $N \gg 1$; ii) The low atomic excitation condition, i.e. the control fields are much stronger than the quantized probe fields and only a few atoms occupy the metastable state $|c\rangle$ and excited states $\left|e_{j}\right\rangle$. It then follows that $\left[\hat{E}_{i}, \hat{E}_{j}^{\dagger}\right]=\delta_{i j}$ and $\left[\hat{C}, \hat{C}^{\dagger}\right]=1$ and all the other commutators are zero, which shows the mutual independence between these bosonic operators $\hat{E}_{\sigma}$ and $\hat{C}$. On the 


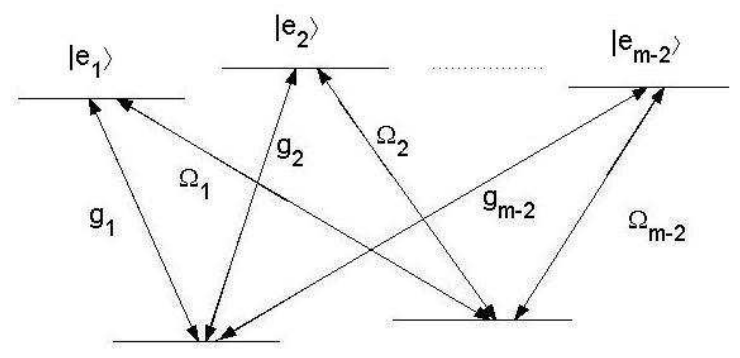

|b)

$|c\rangle$

(a)

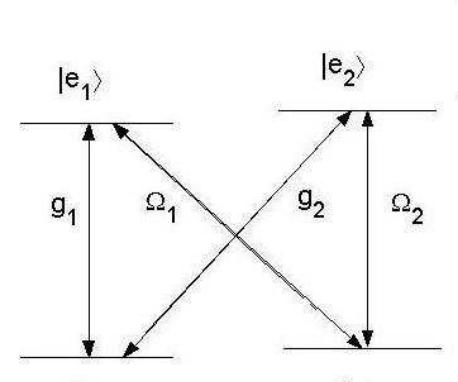

$|b\rangle$

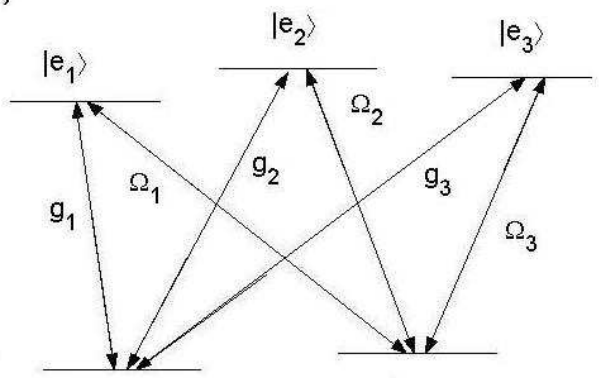

|b)

$|c\rangle$

(b)

(c)

Figure 1: Single ensemble composed of multi-level atoms interacts with with many single-mode quantized and classical control fields. In particular, (a) General $m$-level atomic-ensemble; (b) Four-level case; (c) Five-level $W$ type atom-ensemble case.

other hand, the $m^{2}-3 m+2$ collective operators $\hat{T}_{\mu \nu}$ satisfy the $u(m-1)$ commutation relation: $\left[\hat{T}_{\alpha \beta}, \hat{T}_{\mu \nu}\right]=\delta^{\beta \mu} \hat{T}_{\alpha \nu}-\delta^{\alpha \nu} \hat{T}_{\mu \beta}$. Thus the operators $\left(\hat{T}_{\mu \nu}^{ \pm}, \hat{T}_{\mu \nu}^{z}\right)\left(\mu, \nu=c, e_{1}, e_{2}, \ldots, e_{m-2}\right)$ compose the $m^{2}-2 m+1$ generators of the algebra $s u(m-1)$, here

$$
\hat{T}_{\mu \nu}^{z}=\sum_{j=1}^{N}\left(\hat{\sigma}_{\mu \mu}^{j}-\hat{\sigma}_{\nu \nu}^{j}\right) / 2,\left(\mu \neq \nu=c, e_{1}, e_{2}, \ldots, e_{m-2}\right),
$$

with the relation $\hat{T}_{\mu \nu}^{z}=\hat{T}_{\mu \rho}^{z}-\hat{T}_{\rho \nu}^{z}$. Considering $\left[\hat{T}_{c e_{\sigma}}^{+}, \hat{E}_{\sigma}\right]=-\hat{C},\left[\hat{T}_{c e_{\sigma}}^{-}, \hat{C}\right]=-\hat{E}_{\sigma},\left[\hat{T}_{e_{i} e_{j}}^{+}, \hat{E}_{k}\right]=\delta_{j k} \hat{E}_{i}-$ $\delta_{i k} \hat{E}_{j},\left[\hat{T}_{e_{i} e_{j}}^{-}, \hat{E}_{k}\right]=\delta_{i k} \hat{E}_{j}-\delta_{j k} \hat{E}_{i}$ and denoting by $h_{m-1}$ the algebra generated by $\left(\hat{E}_{\sigma}, \hat{E}_{\sigma}^{\dagger}, \hat{C}, \hat{C}^{\dagger}\right)$, we then obtain $\left[s u(m-1), h_{m-1}\right] \subset h_{m-1}$ which means that the dynamical symmetry of the $m$-level atomic system is governed by a semidirect product Lie group [12] $S U(m-1) \bar{\otimes} H_{m-1}$ in large $N$ limit and low excitation condition. In general, the dynamical symmetry of a $m$-level atomic system is governed by $S U(m)$ [], e.g. the Gell-Mann dynamical symmetry $S U(3)$ for three-level quantum system [13. However, here in the large atom number limit and low excitation condition, the dynamical symmetry of the multi-level EIT system is governed by a semi-direct Lie group. Particularly, when $m=3$, i.e., for the usual three-level system, the dynamical symmetry is governed by the simplest $S U(2) \bar{\otimes} H_{2}$ group [8], while the four-level double $\Lambda(m=4)$ system [14, 15] is governed by $S U(3) \bar{\otimes} H_{3}$ [9] (Fig. 1(b)) and the five-level $W$-type system governed by $S U(4) \bar{\otimes} H_{4}$ (Fig. 1(c)), etc.. 


\subsection{Multi-atomic-ensemble system of three-level atoms}

In this subsection we consider a cloud of identical atoms with the three-level $\Lambda$ type structure which is shown in Fig. 2. Atoms of the $l$-th $(l=1,2, \ldots k)$ atomic ensemble interact with the input singlemode quantized field with coupling constants $g_{l}$, and one classical control filed with time-dependent Rabi-frequencies $\Omega_{l}(t)$. Considering all transitions at resonance, the interaction Hamiltonian of the total system can be written as:
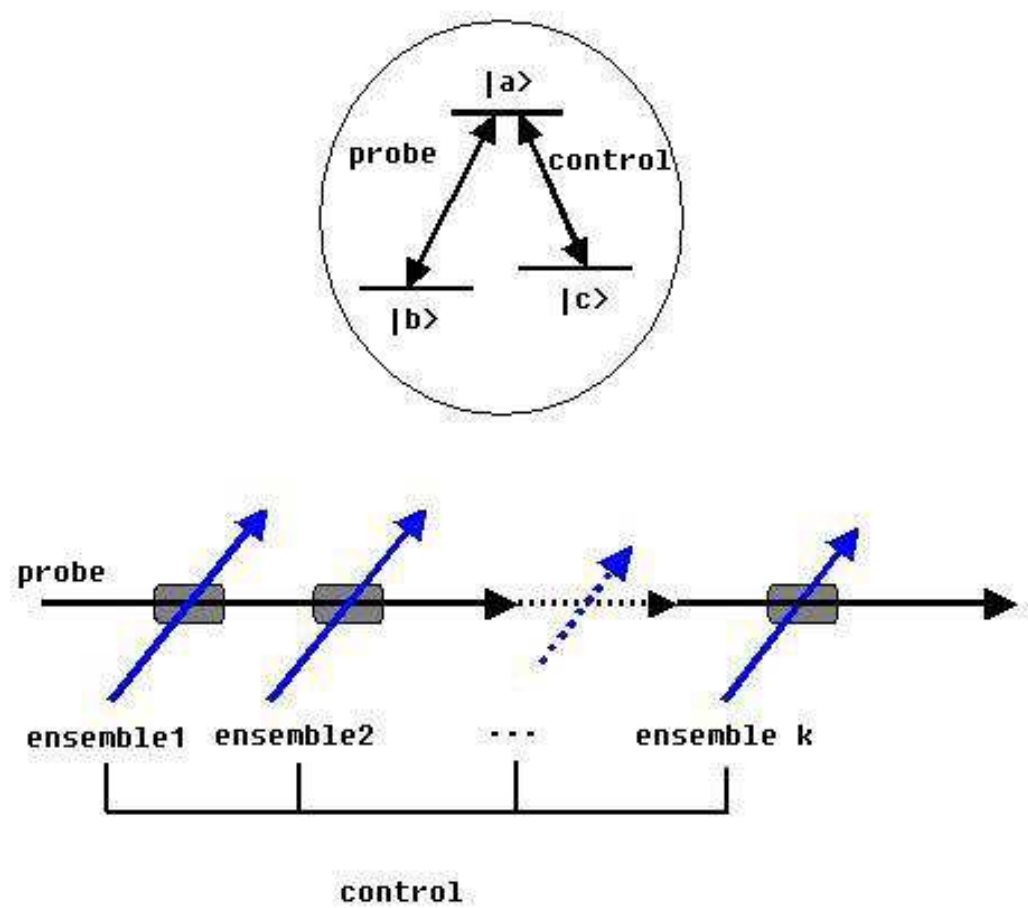

Figure 2: (color online) EIT process for many ensembles composed of $\Lambda$ type atoms located in the straight-line configuration.

$$
\hat{H}=\sum_{\sigma=1}^{k} g_{\sigma} \sqrt{N_{\sigma}} \hat{a} \hat{A}_{\sigma}^{\dagger}+\sum_{\sigma=1}^{k} \Omega_{\sigma}(t) \hat{T}_{\sigma}^{+}+h . c .
$$

where the subscript $\sigma$ denotes the corresponding atomic ensemble and the collective atomic excitation operators:

$$
\hat{A}_{\sigma}=\frac{1}{\sqrt{N_{\sigma}}} \sum_{j=1}^{N_{\sigma}} e^{-i \mathbf{k}_{\mathbf{b a}} \cdot \mathbf{r}_{\mathbf{j}}^{(\sigma)}} \hat{\sigma}_{b a}^{j(\sigma)}, \quad \hat{C}_{\sigma}=\frac{1}{\sqrt{N_{\sigma}}} \sum_{j=1}^{N_{\sigma}} e^{-i \mathbf{k}_{\mathbf{b c}} \cdot \mathbf{r}_{\mathbf{j}}^{(\sigma)}} \hat{\sigma}_{b c}^{j(\sigma)}, \quad \sigma=1,2, \ldots, k
$$

with $\hat{\sigma}_{\mu \nu}^{i}=|\mu\rangle_{i i}\langle\nu|(\mu, \nu=a, b, c)$ being the flip operators of the $i$-th atom between states $|\mu\rangle$ and $|\nu\rangle$, $\mathbf{k}_{\mathbf{b a}}$ and $\mathbf{k}_{\mathbf{c a}}$ are, respectively, the wave vectors of the quantum and classical light fields, $\mathbf{k}_{\mathbf{b c}}=\mathbf{k}_{\mathbf{b a}}-\mathbf{k}_{\mathbf{c a}}$ and

$$
\hat{T}_{\sigma}^{-}=\left(\hat{T}_{\sigma}^{+}\right)^{\dagger}=\sum_{j=1}^{N_{\sigma}} e^{-i \mathbf{k}_{\mathrm{ca}} \cdot \mathbf{r}_{\mathbf{j}}^{(\sigma)}} \hat{\sigma}_{c a}^{j(1)} .
$$


Denoting by $\left|b^{(\sigma)}\right\rangle=\left|b_{1}^{(\sigma)}, b_{2}^{(\sigma)}, \ldots, b_{N_{\sigma}}^{(\sigma)}\right\rangle(\sigma=1,2, \ldots, k)$ the collective ground state of the $\sigma$-th atomic ensemble with all atoms staying in the same single particle ground state $|b\rangle$, we can easily give other quasi-spin wave states by the operators defined in formula (11): $\left|a_{(\sigma)}^{n}\right\rangle=[n !]^{-1 / 2}\left(\hat{A}_{\sigma}^{\dagger}\right)^{n}\left|b^{(\sigma)}\right\rangle$ and $\left|c_{(\sigma)}^{n}\right\rangle=[n !]^{-1 / 2}\left(\hat{C}_{\sigma}^{\dagger}\right)^{n}\left|b^{(\sigma)}\right\rangle$. Similarly, in large $N_{\sigma}$ limit and low excitation condition, it follows that $\left[\hat{A}_{(i)}, \hat{A}_{(j)}^{\dagger}\right]=\delta_{i j},\left[\hat{C}_{(i)}, \hat{C}_{(j)}^{\dagger}\right]=\delta_{i j}$ and all the other commutators are zero, which shows the mutual independence between these bosonic operators $\hat{A}_{i}$ and $\hat{C}_{i}$. On the other hand, one can easily find the commutation relations: $\left[\hat{T}_{i}^{+}, \hat{T}_{j}^{-}\right]=\delta_{i j} \hat{T}_{j}^{z}$ and $\left[\hat{T}_{i}^{z}, \hat{T}_{j}^{ \pm}\right]= \pm \delta_{i j} \hat{T}_{j}^{ \pm}$, where

$$
\hat{T}_{\sigma}^{z}=\sum_{j=1}^{N_{\sigma}}\left(e^{-i \mathbf{k}_{\mathbf{a a}} \cdot \mathbf{r}_{\mathbf{j}}^{(\sigma)}} \hat{\sigma}_{a a}^{j(\sigma)}-e^{-i \mathbf{k}_{\mathbf{c c}} \cdot \mathbf{r}_{\mathbf{j}}^{(\sigma)}} \hat{\sigma}_{c c}^{j(\sigma)}\right) / 2(\sigma=1,2, \ldots, m)
$$

are two traceless operators. Thus the operators $\left(\hat{T}_{\sigma}^{ \pm}, \hat{T}_{\sigma}^{z}\right)$ generate the $\oplus_{\sigma} s u(2)$ algebra. Considering $\left[\hat{T}_{i}^{+}, \hat{A}_{j}\right]=-\delta_{i j} \hat{C}_{j},\left[\hat{T}_{i}^{-}, \hat{C}_{j}\right]=-\delta_{i j} \hat{A}_{j}$ and denoting by $h_{2 m}$ the Heisenberg algebra generated by $\left(\hat{A}_{i}, \hat{A}_{i}^{\dagger}, \hat{C}_{i}, \hat{C}_{i}^{\dagger} ; i=1,2, \ldots, k\right)$, we then obtain $\left[\oplus_{\sigma} s u(2), h_{2 k}\right] \subset h_{2 k}$ which means that the dynamical symmetry of the double $\Lambda$ system is governed by a semidirect product Lie group [12] $\left(\otimes_{\sigma} S U(2)\right) \bar{\otimes} H_{2 k}$ in large $N_{\sigma}$ limit and low excitation condition. In particular, for $k=2$, the symmetrical group reads $S O(4)) \bar{\otimes} H_{4}[10$.

\section{Quantum memory process in multi-level and multi-ensemble atomic system}

The discovery of dynamical symmetry in above section leads us, by the spectrum generating algebra method [12], to find $H$-invariant subspaces, in which one can diagonalize the Hamiltonian easily. As is known, the zero-eigenvalue subspace composed of dark states is the key definition in quantum memory with EIT technique [5, 6, 7, 8]. During the quantum memory process when the quantum states are adiabatically transferred from lights to collective atom coherence, the total system of the atoms and quantized probe light should be restricted in the dark-state subspace [5, 6, 7, 8, therefore the key point of studying this process is to obtain the dark states of the total system. On the other hand, the dark states can be generated by the dark-state polaritons (DSPs) operator which commutes with the Hamiltonian operator [5, 6, 8], so we firstly study the general definition of the DSPs operator in the general multi-level atomic and multi-ensemble atomic systems, and can then easily study the quantum memory process by generating the dark states of the system.

DSPs operator can be constructed basing on two properties: 1) It commutes with the Hamiltonian and satisfies the bosonic commutation relation; 2) It is the superposition of the collective atomic excitation operator $\hat{C}$ and the annihilation operators of the quantized probe lights. For this the dark-state subspace is a collection composed of zero-eigenstates excluding any excited state $\left|e_{j}\right\rangle$ (in multi-level system) or $|a\rangle$ (in multi-atomic-ensemble system). For the derivation of the DSPs operator, firstly we can obtain its form of the three-level Lambda system [8], and four-level double Lambda system [9], and also the five-level case. Then, by induction we can obtain the general definition of the DSPs operator in m-level case, which is similar to that in multi-atomic-ensemble system. 


\subsection{Quantum memory with a $m$-level atomic system}

Firstly, we study the general definition of DSPs of the single-atomic-ensemble system with many $m$-level atoms. Based on the above analysis of the properties of DSP operator, the new type of dark-state-polaritons operator of the $m$-level system can be defined as

$$
\hat{d}=\cos \theta \prod_{j=1}^{m-3} \cos \phi_{j} \hat{a}_{1}+\cos \theta \sum_{l=2}^{m-2} \sin \phi_{l-1} \prod_{j=l}^{m-3} \cos \phi_{j} \hat{a}_{l}-\sin \theta \hat{C},
$$

where the mixing angles $\theta$ and $\phi_{j}$ are defined through

$$
\tan \theta=\frac{g_{1} g_{2} \ldots g_{m-2} \sqrt{N}}{\left[\sum_{j=1}^{m-2}\left(\Omega_{j}^{2} \prod_{l=1, l \neq j}^{m-2} g_{l}^{2}\right)\right]^{1 / 2}}
$$

and

$$
\tan \phi_{j}=\frac{\prod_{l=1}^{j} g_{l} \Omega_{j+1}}{\left[\sum_{l=1}^{j}\left(\Omega_{l}^{2} \prod_{s=1, s \neq l}^{j+1} g_{s}^{2}\right)\right]^{1 / 2}} .
$$

The eq. (11) provides us $\tan \phi_{1}=g_{1} \Omega_{2} / g_{2} \Omega_{1}, \tan \phi_{2}=g_{1} g_{2} \Omega_{3} / \sqrt{\Omega_{1}^{2} g_{2}^{2} g_{3}^{2}+\Omega_{2}^{2} g_{1}^{2} g_{3}^{2}} \ldots$, etc. By a straightforward calculation one can verify that

$$
\left[\hat{d}, \hat{d}^{\dagger}\right]=1, \quad[\hat{H}, \hat{d}]=0,
$$

hence the general atomic dark states of $m$-level system can be obtained through $\left|D_{n}\right\rangle=[n !]^{-1 / 2}\left(\hat{d}^{\dagger}\right)^{n}|0\rangle$, where

$$
|0\rangle=\underbrace{\mid 0,0, \ldots, 0}_{m-2}\rangle_{\text {photon }} \otimes|b\rangle_{\text {atom }}
$$

are the collective ground states [16] with $|0,0, \ldots, 0\rangle_{\text {photon }}$ denoting the electromagnetic vacuum of $m-2$ quantized probe fields.

Based on the above result we here discuss a novel phenomenon. Initially, only one weak probe light (described by the coherent state $\left|\alpha_{1}\right\rangle$ with $\alpha_{1}=\alpha_{0}$ ) is injected into the atomic ensemble to couple the transition from $|b\rangle$ to $\left|e_{1}\right\rangle$, one strong control field is used to couple the transition from $|c\rangle$ to $\left|e_{1}\right\rangle$ and all other light fields ( $m-3$ probe fields and $m-3$ control fields) are off. For this the mixing angles $\theta=0, \phi_{j}=0$ and the initial total state of the quantized field and atomic ensemble reads $\left|\Psi_{0}\right\rangle=\sum_{n} P_{n}\left(\alpha_{0}\right)|n, \underbrace{0,0, \ldots, 0}_{m-3}\rangle_{p h o t o n} \otimes|b\rangle_{\text {atom }}$, where $P_{n}\left(\alpha_{0}\right)=\frac{\alpha_{0}^{n}}{\sqrt{n !}} e^{-\left|\alpha_{0}\right|^{2} / 2}$ is the probability of distribution function. Subsequently, the mixing angle $\theta$ is adiabatically rotated to $\pi / 2$ by turning the control field off, and the quantum states of the probe light $\left|\alpha_{1}\right\rangle$ is fully mapped into the collective atomic excitations, i.e. $\left|\Psi_{t}\right\rangle=\sum_{n} P_{n}\left(\alpha_{0}\right)|\underbrace{0,0, \ldots, 0}_{m-2}\rangle_{\text {photon }} \otimes\left|c^{n}\right\rangle_{\text {atom }}$. Finally, when all $m-2$ control fields are all turned back on and the mixing angle $\theta$ is rotated back to $\theta=0$ again with $\phi_{j}$ to some value $\phi_{e j}$ which are only determined by the Rabi-frequencies of the re-applied control fields, we finally obtain

$$
\begin{aligned}
\left|\Psi_{e}\right\rangle & =\sum_{n} P_{n}\left(\alpha_{0}\right)\left|D_{n}(\theta=0)\right\rangle \\
& =\sum_{j} \sum_{l} \ldots \sum_{f} P_{j}\left(\alpha_{e 1}\right) P_{l}\left(\alpha_{e 2}\right) \ldots P_{f}\left(\alpha_{e(m-2)}\right)|b\rangle \otimes|j, l, \ldots, f\rangle \\
& =|b\rangle_{\text {atom }} \otimes\left|\alpha_{e 1}, \alpha_{e 2}, \ldots, \alpha_{e(m-2)}\right\rangle_{\text {photon }},
\end{aligned}
$$


where $\alpha_{e 1}=\alpha_{0} \prod_{j=1}^{m-3} \cos \phi_{e j}$ and $\alpha_{e l}=\alpha_{0} \sin \phi_{e(l-1)} \prod_{j=l}^{m-3} \cos \phi_{e j},(l=2,3, \ldots, m-2)$ are the parameters of the released coherent lights. The above expression clearly shows that the injected quantized field can convert into $m-2$ different coherent pulses $\left|\alpha_{e j}\right\rangle(j=1,2, \ldots, m-2)$ after a proper evolution manipulated by the control fields. Particularly, if the strengths of all re-applied control fields equal each other, the output probe lights read $\alpha_{e 1}=\alpha_{e 2}=\ldots=\alpha_{e(m-2)}=\alpha_{0} / \sqrt{m-2}$. Obviously, this novel mechanism can be extended to other cases of the injected field, say, in presence of a non-classical or squeezed light beam.

\subsection{Quantum memory with k-atomic-ensemble system}

Now, to give a clear description of the interesting quantum memory process in this $k$-atomic-ensemble system composed of $\Lambda$ type three-level-atoms, we define the new type of dark-state-polaritons operator as

$$
\hat{d}=\cos \theta \hat{a}-\sin \theta \prod_{j=1}^{k-1} \cos \phi_{j} \hat{C}_{1}-\sin \theta \sum_{l=2}^{k} \sin \phi_{l-1} \prod_{j=l}^{k-1} \cos \phi_{j} \hat{C}_{l},
$$

where the mixing angles $\theta$ and $\phi_{j}$ are defined through

$$
\tan \theta=\frac{\left[\sum_{j=1}^{k}\left(g_{j}^{2} N_{j} \prod_{l=1, l \neq j}^{k} \Omega_{l}^{2}\right)\right]^{1 / 2}}{\Omega_{1} \Omega_{2} \ldots \Omega_{k}}
$$

and

$$
\tan \phi_{j}=\frac{g_{j+1} \sqrt{N_{j+1}} \prod_{l=1}^{j} \Omega_{l}}{\left[\sum_{l=1}^{j}\left(g_{l}^{2} N_{l} \prod_{s=1, s \neq l}^{j+1} \Omega_{s}^{2}\right]^{1 / 2}\right.},
$$

where one finds $\tan \phi_{1}=g_{2} \sqrt{N_{2}} \Omega_{1} / g_{1} \sqrt{N_{1}} \Omega_{2}, \tan \phi_{2}=g_{3} \sqrt{N_{3}} \Omega_{1} \Omega_{2} / \sqrt{g_{1}^{2} N_{1} \Omega_{2}^{2} \Omega_{3}^{2}+g_{2}^{2} N_{2} \Omega_{1}^{2} \Omega_{3}^{2}}$, etc. Also, by a straightforward calculation one can verify that $\left[\hat{d}, \hat{d}^{\dagger}\right]=1$ and $[\hat{H}, \hat{d}]=0$, hence the general atomic dark states can be obtained through $\left|D_{n}\right\rangle=[n !]^{-1 / 2}\left(\hat{d}^{\dagger}\right)^{n}|0\rangle$, where $|0\rangle=\left|b^{(1)}, b^{(2)}, \ldots, b^{(k)}\right\rangle_{\text {atom }} \otimes$ $|0\rangle_{\text {photon }}$ and $|0\rangle_{\text {photon }}$ denotes the electromagnetic vacuum of the quantized probe field.

Similar to the discussion in above subsection, we can investigate the quantum memory process in the multi-ensemble atomic system. Initially the total state reads (meanwhile $\theta=0$ or the external control fields are very strong): $\left|\Psi_{0}\right\rangle=\sum_{n} P_{n}\left(\alpha_{0}\right)\left|b^{(1)}, b^{(2)}, \ldots, b^{(k)}\right\rangle_{\text {atom }} \otimes|n\rangle_{\text {photon }}$, then the mixing angle $\theta$ is adiabatically rotated from 0 to $\pi / 2$ by keeping the ratio between arbitrary two of the Rabi-frequencies $\Omega_{1}, \Omega_{2} \ldots$ and $\Omega_{k}$ in a fixed value (i.e. keeping the mixing angles $\phi_{j}$ constant) and switching them off adiabatically, we finally obtain the state from the dark-state of present system:

$$
\begin{aligned}
|\Psi(t)\rangle & =\sum_{n} P_{n}\left(\alpha_{0}\right)\left|D_{n}\left(\theta=\frac{\pi}{2}\right)\right\rangle \\
& =\sum_{j} \sum_{l} \ldots \sum_{f} P_{j}\left(\alpha_{1}\right) P_{l}\left(\alpha_{2}\right) \ldots P_{f}\left(\alpha_{k}\right)\left|c_{j}^{(1)}, c_{l}^{(2)}, \ldots, c_{f}^{(k)}\right\rangle \otimes|0\rangle \\
& =\left|\alpha_{1}, \alpha_{2}, \ldots, \alpha_{k}\right\rangle_{\text {coherence }} \otimes|0\rangle_{\text {photon }},
\end{aligned}
$$

where $\alpha_{1}=\alpha_{0} \prod_{j=1}^{k-1} \cos \phi_{j}, \alpha_{l}=\alpha_{0} \sin \phi_{l-1} \prod_{j=l}^{k-1} \cos \phi_{j},(l=2,3, \ldots, k)$. The above expression clearly shows that the injected quantized field can be stored in the $k$ atomic ensembles. Particularly, if the strengths of all control fields keep the same value during the process that they are turned off, the final atom coherence reads $\alpha_{1}=\alpha_{2}=\ldots=\alpha_{k}=\alpha_{0} / \sqrt{k}$, which means the quantum information of the initial probe light is stored homogeneously in the $k$ atomic ensembles. 


\section{Generation of quantum entanglement}

In above section, we discussed the interesting phenomenon that one input coherent probe light can convert into many different output coherent probe lights via the dark-state evolution process. In this section we shall discuss another novel application to the generation of entangled states of lights or atomic ensembles with present DSPs theory in multi-level and multi-ensemble atomic systems. For this we should use a non-classical input probe light, for example, a superposition of coherent states [17, a single-photon state, etc.

\subsection{Two-photon entanglement}

The coherent entangled states can be obtained with the quantized DSPs theory of four-level system when the injected quantized field is in a Schödinger cat state [17, e.g. for the initial total state reads $\left|\Psi_{0}\right\rangle^{ \pm}=\frac{1}{\sqrt{\mathcal{N}\left(\alpha_{0}\right)}}|0\rangle \otimes\left(\left|\alpha_{0}\right\rangle \pm\left|-\alpha_{0}\right\rangle\right) \otimes|b\rangle$ where the normalized factor $\mathcal{N}_{ \pm}\left(\alpha_{0}\right)=2 \pm 2 e^{-2\left|\alpha_{0}\right|^{2}}$ with the same process discussed in the section III.A (see eq. (14), set $m=4$ ) we find the injected quantized pulse can evolve into a very interesting entangled coherent state (ECS) of two output fields $\left(\left|\Psi_{0}\right\rangle^{ \pm} \rightarrow\left|\Psi_{e}\right\rangle^{ \pm}\right)$

$$
\begin{aligned}
& \frac{1}{\sqrt{\mathcal{N}_{ \pm}\left(\alpha_{0}\right)}}|0\rangle \otimes\left(\left|\alpha_{0}\right\rangle \pm\left|-\alpha_{0}\right\rangle\right) \otimes|b\rangle=\frac{1}{\sqrt{\mathcal{N}_{ \pm}\left(\alpha_{0}\right)}}\left(|0\rangle \otimes\left|\alpha_{0}\right\rangle \pm|0\rangle \otimes\left|-\alpha_{0}\right\rangle\right) \otimes|b\rangle \longrightarrow \\
& \longrightarrow \frac{1}{\sqrt{\mathcal{N}_{ \pm}\left(\alpha_{0}\right)}}\left(\sum_{j} \sum_{k} P_{j}\left(\alpha_{e 1}\right) P_{k}\left(\alpha_{e 2}\right)|b, j, k\rangle \pm \sum_{j} \sum_{k} P_{j}\left(-\alpha_{e 1}\right) P_{k}\left(-\alpha_{e 2}\right)|b, j, k\rangle\right) .
\end{aligned}
$$

The final state in above formula can be rewritten as:

$$
\left|\Psi_{e}\right\rangle^{ \pm}=\frac{1}{\sqrt{\mathcal{N}_{ \pm}\left(\alpha_{0}\right)}}\left(\left|\alpha_{e 1}, \alpha_{e 2}\right\rangle \pm\left|-\alpha_{e 1},-\alpha_{e 2}\right\rangle\right)_{\text {photon }} \otimes|b\rangle .
$$

If $\phi_{e}=0$, hence $\alpha_{e 1}=\alpha_{0}$ and $\alpha_{e 2}=0$, and then the evolution of the quantized fields proceed as $|0\rangle \otimes\left(\left|\alpha_{0}\right\rangle \pm\left|-\alpha_{0}\right\rangle\right) / \sqrt{\mathcal{N}_{ \pm}\left(\alpha_{0}\right)} \rightarrow\left(\left|\alpha_{0}\right\rangle \pm\left|-\alpha_{0}\right\rangle\right) \otimes|0\rangle / \sqrt{\mathcal{N}_{ \pm}\left(\alpha_{0}\right)}$, which means the input Schödinger cat state is now fully converted into another one with different vibrational mode. On the other hand, for the general case of non-zero value of the coherent parameters $\alpha_{e 1}$ and $\alpha_{e 2}$, the states of output quantized fields are entangled coherent states. Since the parameters $\alpha_{e i}(i=1,2)$ is controllable, the entanglement of the output states [18] $E^{ \pm}\left(\alpha_{e 1}, \alpha_{e 2}\right)=-\operatorname{tr}\left(\rho_{\alpha_{e 1}}^{ \pm} \ln \rho_{\alpha_{e 1}}^{ \pm}\right)$with the reduced density matrix $\rho_{\alpha_{e 1}}^{ \pm}=\operatorname{tr}^{\left(\alpha_{e 2}, a t o m\right)}\left(\left|\Psi_{e}\right\rangle\left\langle\Psi_{e}\right|\right)^{ \pm}$can also easily be controlled by the re-applied control fields. In particular, for the initial state $\left|\Psi_{0}\right\rangle^{-}$, if $\phi_{e}=\pi / 4$, we have $\alpha_{e 1}=\alpha_{e 2}=\alpha_{0} / \sqrt{2}$ and then obtain the maximally entangled state(MES): $|0\rangle \otimes\left(\left|\alpha_{0}\right\rangle-\left|-\alpha_{0}\right\rangle\right) / \sqrt{\mathcal{N}_{-}\left(\alpha_{0}\right)} \rightarrow\left(\left|\frac{\alpha_{0}}{\sqrt{2}}, \frac{\alpha_{0}}{\sqrt{2}}\right\rangle-\right.$ $\left.\left|-\frac{\alpha_{0}}{\sqrt{2}},-\frac{\alpha_{0}}{\sqrt{2}}\right\rangle\right) / \sqrt{\mathcal{N}_{-}\left(\alpha_{0}\right)}$ which is most useful for quantum information process. With the definitions $|+\rangle=\left(\left|\frac{\alpha_{0}}{\sqrt{2}}\right\rangle+\left|-\frac{\alpha_{0}}{\sqrt{2}}\right\rangle\right) / \sqrt{\mathcal{N}_{+}\left(\alpha_{0} / 2\right)}$ and $|-\rangle=\left(\left|\frac{\alpha_{0}}{\sqrt{2}}\right\rangle-\left|-\frac{\alpha_{0}}{\sqrt{2}}\right\rangle\right) / \sqrt{\mathcal{N}_{-}\left(\alpha_{0} / 2\right)}$, the output state can be rewritten as $(|+\rangle|-\rangle+|-\rangle|+\rangle) / \sqrt{2}$ which is the maximum entangled state of output light pulses. Generalization of these results to multi-mode probe pulses is straightforward. Since our scheme of generating the entangled coherent states via quantized DSPs theory is linear and controllable and it only requires a macroscopic quantum superposition for the initial state, this scheme deserves study in experiment which has made much progress recent years [19]. Remarkably, the latest works have reported the experimental realization of EIT quantum memory in three-level system[20. For our scheme, the key point in experiment is to store the quantum states of one non-classical probe light in a multi-level system, e.g. a four-level system, and then use two control fields to convert quantum states of 
the initial probe light into an entangled state of two output pulses. Since the quantum memory for few probe photons is experimentally realized in three-level system, our scheme of generating entanglement of photons via multi-level system may be reached in near future. Also, our scheme is different from those schemes of generating entangled coherent states via Kerr effect [21] and entanglement swapping using Bell-state measurement [22, which are very important and have been widely studied.

Consider now a different type of input quantum state corresponding to a single-photon state, i.e. meanwhile the initial total state

$$
\left|\Psi_{0}\right\rangle=(|0\rangle \otimes|1\rangle)_{\text {photon }} \otimes|b\rangle
$$

Similarly, after the light state storage and release process discussed above, one can easily obtained the final entangled states of two probe photons:

$$
\Phi_{\text {photon }}=\frac{1}{\sqrt{2}}(|1\rangle|0\rangle+|0\rangle|1\rangle)_{\text {photon }} .
$$

Also, if the input quantum state corresponding to a multi-photon state, we can obtain many other entangled forms of the two output probe lights.

\subsection{Three-photon entanglement via five-level EIT}

Here we consider the similar case that the injected quantized field is in a Schödinger cat state, e.g., meanwhile from the eq. (14) (set $m=5)$ the initial total state reads $\left|\Psi_{0}\right\rangle^{ \pm}=\frac{1}{\sqrt{\mathcal{N}\left(\alpha_{0}\right)}}|0,0\rangle \otimes\left(\left|\alpha_{0}\right\rangle \pm\right.$ $\left.\left|-\alpha_{0}\right\rangle\right) \otimes|b\rangle$ where the normalized factor $\mathcal{N}_{ \pm}\left(\alpha_{0}\right)=2 \pm 2 e^{-2\left|\alpha_{0}\right|^{2}}$, with the similar process used for two-photon entanglement generation we find the injected quantized pulse can evolve into the very interesting entangled coherent states (ECS) of three output fields $\left(\left|\Psi_{0}\right\rangle^{ \pm} \rightarrow\left|\Psi_{e}\right\rangle^{ \pm}\right)$

$$
\begin{gathered}
\frac{1}{\sqrt{\mathcal{N}_{ \pm}\left(\alpha_{0}\right)}}|0,0\rangle \otimes\left(\left|\alpha_{0}\right\rangle \pm\left|\beta_{0}\right\rangle\right) \otimes|b\rangle \rightarrow \\
\longrightarrow \frac{1}{\sqrt{\mathcal{N}_{ \pm}\left(\alpha_{0}\right)}}\left(\left|\alpha_{e 1}, \alpha_{e 2}, \alpha_{e 3}\right\rangle \pm\left|\beta_{e 1}, \beta_{e 2}, \beta_{e 3}\right\rangle\right)_{p h o t o n} \otimes|b\rangle,
\end{gathered}
$$

where $\alpha_{e 1}=\cos \phi \cos \varphi \alpha_{0}, \alpha_{e 2}=\sin \phi \cos \varphi \alpha_{0}, \alpha_{e 3}=\sin \varphi \alpha_{0}$ and $\beta_{e 1}=\cos \phi \cos \varphi \beta_{0}, \beta_{e 2}=\sin \phi \cos \varphi \beta_{0}$ and $\beta_{e 3}=\sin \varphi \beta_{0}$. If $\phi=\pi / 4$ and $\varphi=\tan ^{-1} \frac{\sqrt{2}}{2}$, we get $\alpha_{e j}=\alpha=\alpha_{0} / \sqrt{3}, \beta_{e j}=\beta=\beta_{0} / \sqrt{3}(j=$ $1,2,3)$, and the final state of the atom coherence: $(|\alpha, \alpha, \alpha\rangle \pm|\beta, \beta, \beta\rangle)_{\text {photon }} / \sqrt{\mathcal{N}_{0 \pm}}$. With the definitions $|+\rangle=\left(\left|\frac{\alpha_{0}}{\sqrt{2}}\right\rangle+\left|-\frac{\alpha_{0}}{\sqrt{2}}\right\rangle\right) / \sqrt{\mathcal{N}_{+}\left(\alpha_{0} / \sqrt{3}\right)}$ and $|-\rangle=\left(\left|\frac{\alpha_{0}}{\sqrt{2}}\right\rangle-\left|-\frac{\alpha_{0}}{\sqrt{2}}\right\rangle\right) / \sqrt{\mathcal{N}_{-}\left(\alpha_{0} / \sqrt{3}\right)}$, the output state can be rewritten as

$$
\Phi_{\text {photon }}(+)=\frac{1}{\sqrt{\mathcal{N}_{0+}}}\left(\left|\frac{\alpha_{0}}{\sqrt{3}}, \frac{\alpha_{0}}{\sqrt{3}}, \frac{\alpha_{0}}{\sqrt{3}}\right\rangle+\left|\frac{\beta_{0}}{\sqrt{3}}, \frac{\beta_{0}}{\sqrt{3}}, \frac{\beta_{0}}{\sqrt{3}}\right\rangle\right)_{\text {photon }}=h_{1}|+\rangle|+\rangle|+\rangle+h_{2}\left|W_{+}\right\rangle
$$

and

$$
\Phi_{\text {photon }}(-)=\frac{1}{\sqrt{\mathcal{N}_{0-}}}\left(\left|\frac{\alpha_{0}}{\sqrt{3}}, \frac{\alpha_{0}}{\sqrt{3}}, \frac{\alpha_{0}}{\sqrt{3}}\right\rangle-\left|\frac{\beta_{0}}{\sqrt{3}}, \frac{\beta_{0}}{\sqrt{3}}, \frac{\beta_{0}}{\sqrt{3}}\right\rangle\right)_{\text {photon }}=h_{1}^{\prime}|-\rangle|-\rangle|-\rangle+h_{2}^{\prime}\left|W_{-}\right\rangle,
$$

where $\left|W_{+}\right\rangle=|+\rangle|-\rangle|-\rangle+|-\rangle|+\rangle|-\rangle+|-\rangle|-\rangle|+\rangle$and $\left|W_{-}\right\rangle=|-\rangle|+\rangle|+\rangle+|+\rangle|-\rangle|+\rangle+|+\rangle|+\rangle|-\rangle$are $W$ states [23] of three light fields. $h_{1}=\sqrt{N_{+} N_{-}^{2} / 16 N_{0+}^{2}}, h_{1}^{\prime}=\sqrt{N_{-} N_{+}^{2} / 16 N_{0-}^{2}}, h_{2}=\sqrt{N_{+}^{3} / 4 N_{0+}^{2}}$ and $h_{2}^{\prime}=\sqrt{N_{-}^{3} / 16 N_{0-}^{2}}$. The eqs.(24) and (25) indicate a fascinating phenomenon: The two-light 
state is still entangled after reducing the third one. Similar to the result of eqs. (21) and (22), when the input probe light is in a single-photon state, one can obtain the maximum entangled states of three-mode photons.

It is noteworthy that the five-qubit code entanglement can be obtained via a seven-level system that interacts with five probe and five control fields, which is the shortest code that can be a error correcting code (ECC) 24]. Furthermore, theoretically the entanglement of $m$ light fields can be obtained using the quantized DSPs theory in multi-level atomic system.

\subsection{Entanglement between two and three atomic ensembles}

Generation of entanglement between atomic ensembles has attracted much attentions in very recent years [25]. Here we also can generate entanglement between atomic ensembles by using multi-atomicensemble EIT technique, which is similar to that in generation of entanglement between coherent lights.

Firstly, one can find that if the injected quantized field is in a Schödinger cat state [17, e.g., for the initial total state reads [10] $\left|\Psi_{0}\right\rangle^{ \pm}=\frac{1}{\sqrt{\mathcal{N}_{ \pm}\left(\alpha_{0}\right)}}\left(\left|\alpha_{0}\right\rangle \pm\left|-\alpha_{0}\right\rangle\right)_{\text {photon }} \otimes\left|b^{(1)}, b^{(2)}\right\rangle_{\text {atom }}$ where the normalized factor $\mathcal{N}_{ \pm}\left(\alpha_{0}\right)=2 \pm 2 e^{-2\left|\alpha_{0}\right|^{2}}$, with the scheme discussed above (see eq. (18), set $k=2$ ) we can finally obtain a very interesting entangled atomic coherence of two atomic ensembles $\left(\left|\Psi_{0}\right\rangle^{ \pm} \rightarrow\left|\Psi_{e}\right\rangle^{ \pm}\right)$

$$
\begin{gathered}
\frac{1}{\sqrt{\mathcal{N}_{ \pm}\left(\alpha_{0}\right)}}\left(\left|\alpha_{0}\right\rangle \pm\left|-\alpha_{0}\right\rangle\right)_{\text {photon }} \otimes\left|b^{(1)}, b^{(2)}\right\rangle_{\text {atom }} \rightarrow \\
\longrightarrow \frac{1}{\sqrt{\mathcal{N}_{ \pm}\left(\alpha_{0}\right)}}|0\rangle_{\text {photon }} \otimes\left(\left|\alpha_{1}, \alpha_{2}\right\rangle \pm\left|-\alpha_{1},-\alpha_{2}\right\rangle\right)_{\text {coherence }} .
\end{gathered}
$$

Particularly, for the initial state $\left|\Psi_{0}\right\rangle^{-}$, if $\phi=\pi / 4$, we have $\alpha_{1}=\alpha_{2}=\alpha_{0} / \sqrt{2}$ and then obtain the maximally entangled state (MES): $(|+\rangle|-\rangle+|-\rangle|+\rangle)$ coherence $/ \sqrt{2}$, where $|+\rangle=\left(\left|\frac{\alpha_{0}}{\sqrt{2}}\right\rangle+\mid-\right.$ $\left.\left.\frac{\alpha_{0}}{\sqrt{2}}\right\rangle\right)_{\text {coherence }} / \sqrt{\mathcal{N}_{+}\left(\alpha_{0} / 2\right)}$ and $|-\rangle=\left(\left|\frac{\alpha_{0}}{\sqrt{2}}\right\rangle-\left|-\frac{\alpha_{0}}{\sqrt{2}}\right\rangle\right)_{\text {coherence }} / \sqrt{\mathcal{N}_{-}\left(\alpha_{0} / 2\right)}$ are the orthogonal basis.

Secondly, the three-atomic-ensemble entanglement can easily obtained for the case of $m=3$. Considering the Schödinger cat state of the injected probe field, for example, if $\left|\Psi_{0}\right\rangle^{ \pm}=\frac{1}{\sqrt{\mathcal{N}_{0 \pm}}}\left(\left|\alpha_{0}\right\rangle \pm\right.$ $\left.\left|\beta_{0}\right\rangle\right)_{\text {photon }} \otimes\left|b^{(1)}, b^{(2)}, b^{(3)}\right\rangle_{\text {atom }}$ with the normalized factor $\mathcal{N}_{0 \pm}=2 \pm 2 e^{-\left|\alpha_{0}-\beta_{0}\right|^{2} / 2}$, the entangled quasi spin-waves between 3 -atomic ensembles can be obtained by properly steering the external control fields

$$
\begin{aligned}
& \frac{1}{\sqrt{\mathcal{N}_{0 \pm}}}\left(\left|\alpha_{0}\right\rangle \pm\left|\beta_{0}\right\rangle\right)_{\text {photon }} \otimes\left|b^{(1)}, b^{(2)}, b^{(3)}\right\rangle_{\text {atom }} \rightarrow \\
\longrightarrow & \frac{1}{\sqrt{\mathcal{N}_{0 \pm}}}|0\rangle_{\text {photon }} \otimes\left(\left|\alpha_{1}, \alpha_{2}, \alpha_{3}\right\rangle \pm\left|\beta_{1}, \beta_{2}, \beta_{3}\right\rangle\right)_{\text {coherence }}
\end{aligned}
$$

where $\alpha_{1}=\cos \phi \cos \varphi \alpha_{0}, \alpha_{2}=\sin \phi \cos \varphi \alpha_{0}, \alpha_{3}=\sin \varphi \alpha_{0}$ and $\beta_{1}=\cos \phi \cos \varphi \beta_{0}, \beta_{2}=\sin \phi \cos \varphi \beta_{0}$ and $\beta_{3}=\sin \varphi \beta_{0}$. Similar to the eqs. (24) and (25), the final entangled states can then be rewritten as

$$
\Phi_{123}(+)=\frac{1}{\sqrt{\mathcal{N}_{0+}}}(|\alpha, \alpha, \alpha\rangle+|\beta, \beta, \beta\rangle)_{\text {coherence }}=h_{1}|+\rangle|+\rangle|+\rangle+h_{2}\left|W_{+}\right\rangle
$$

and

$$
\Phi_{123}(-)=\frac{1}{\sqrt{\mathcal{N}_{0-}}}(|\alpha, \alpha, \alpha\rangle-|\beta, \beta, \beta\rangle)_{\text {coherence }}=h_{1}^{\prime}|-\rangle|-\rangle|-\rangle+h_{2}^{\prime}\left|W_{-}\right\rangle
$$


where the coefficients $h_{1,2}$ and $h_{1,2}^{\prime}$ has the same form as that in eq. (24) and (25), and $\left|W_{ \pm}\right\rangle$are the corresponding $W$ states. Furthermore, theoretically one can generate entangled atomic states between multi-atomic ensembles by extending present results to $m$-atomic-ensemble system.

The above results show many similar features between multi-level systems and multi-ensemble system. In fact, in present large number atoms and weak excitation case, the collective atomic operators satisfy the same commutation relations with the photonic boson operators $\left(\hat{a}_{j}, \hat{a}_{j}^{\dagger}\right)$. Therefore, we can readily conclude a general understanding of the processes that a quantized probe field can be transferred into many probe ones in multi-level system and can be transferred into many ensembles of atomic coherence, say, the process can be generally regarded that a bosonic field can be transferred into many different bosonic ones via EIT quantum memory technique. This may be the basis that we can use multi-level system to generate multi-photon entanglement and use many-ensemble system to generate multi-atomic-ensemble entanglement.

Before conclusion, we should emphasize again the adiabatic condition in the EIT quantum memory process with multi-level and multi-ensemble atomic systems. As we have known, the condition of adiabatic evolution is most important for the quantum memory technique based on the quantized DSPs theory, because the total system should be confined in dark-state subspace during the process of quantum memory. It is interesting that the symmetrical properties of the multi-level system and multi-atomic-ensemble system are dependent on parameters such as the coupling constant $g_{i}$ and Rabi frequency $\Omega_{i}$ etc. For multi-level system, the largest zero-degeneracy class besides dark-state subspace will exist for the case $g_{1}=g_{2}=\ldots=g_{m-2}$ [9, while for the multi-ensemble atomic system, it will do when $\Omega_{1}=\Omega_{2}=\ldots=\Omega_{k}$ [10]. For example, we can give a brief discussion on the five-level system which has the largest degeneracy class when the parameters satisfy $g_{1}=g_{2}=g_{3}=g$. For this we define

$$
\begin{aligned}
\hat{u} & =\cos \phi \hat{E}_{1}+\sin \phi \hat{E}_{2}, \quad \hat{v}=-\sin \phi \hat{E}_{1}+\cos \phi \hat{E}_{2} ; \\
\hat{s} & =\cos \varphi \hat{u}+\sin \varphi \hat{E}_{3}, \quad \hat{f}=-\sin \varphi \hat{u}+\cos \varphi \hat{E}_{3} ; \\
\hat{a}_{12+} & =\cos \phi \hat{a}_{1}+\sin \phi \hat{a}_{2}, \quad \hat{a}_{12-}=-\sin \phi \hat{a}_{1}+\cos \phi \hat{a}_{2} ; \\
\hat{a}_{123+} & =\cos \varphi \hat{a}_{12+}+\sin \varphi \hat{a}_{3}, \quad \hat{a}_{123-}=-\sin \varphi \hat{a}_{12+}+\cos \varphi \hat{a}_{3}
\end{aligned}
$$

and the BSPs operator $\hat{b}=\sin \theta \hat{a}_{123+}+\cos \theta \hat{C}$. Using these definitions one can find the shift operators as follow

$$
\hat{Q}_{ \pm}^{\dagger}=\cos \phi \hat{s}^{\dagger} \pm \sin \phi \hat{b}^{\dagger}, \quad \hat{P}_{ \pm}^{\dagger}=\hat{v}^{\dagger} \pm \hat{a}_{12-}^{\dagger}, \quad \hat{O}_{ \pm}^{\dagger}=\hat{f}^{\dagger} \pm \hat{a}_{123-}^{\dagger},
$$

which satisfy the commutation relations $\left[\hat{H}, \hat{Q}_{ \pm}^{\dagger}\right]= \pm \epsilon_{1} \hat{Q}_{ \pm}^{\dagger}, \quad\left[\hat{H}, \hat{P}_{ \pm}^{\dagger}\right]= \pm \epsilon_{2} \hat{P}_{ \pm}^{\dagger}, \quad\left[\hat{H}, \hat{O}_{ \pm}^{\dagger}\right]= \pm \epsilon_{3} \hat{O}_{ \pm}^{\dagger}$, where $\epsilon_{1}=\sqrt{g^{2} N+\Omega_{1}^{2}+\Omega_{2}^{2}+\Omega_{3}^{2}}$ and $\epsilon_{2}=\epsilon_{3}=g \sqrt{N}$. Thanks to these results we finally obtain the largest degeneracy class of present system:

$$
|r(i, j ; k, l ; f, g ; n)\rangle=\frac{1}{\sqrt{i ! j ! k ! l ! f ! g !}}\left(\hat{Q}_{+}^{\dagger}\right)^{i}\left(\hat{Q}_{-}^{\dagger}\right)^{j}\left(\hat{P}_{+}^{\dagger}\right)^{k}\left(\hat{P}_{-}^{\dagger}\right)^{l}\left(\hat{O}_{+}^{\dagger}\right)^{f}\left(\hat{O}_{-}^{\dagger}\right)^{g}\left|D_{n}\right\rangle
$$

with eigenvalue $E(i, j ; k, l ; f, g)=(i-j) \epsilon_{1}+[(k+f)-(l+g)] \epsilon_{2}$. We notice that for each given pair of indices $(i, j)$ and $(k+f, l+g),\{|r(i, j ; k, l ; f, g ; n)\rangle \mid n=0,1,2, \cdots\}$ defines a degenerate set of eigenstates. When $i=j$ and $k+f=l+g=m, E(i, i ; k+f=l+g)=0$, and a larger zero-eigenvalue 
degeneracy class is given by: $\{|r(i, i ; k, l ; m-k, m-l ; n)\rangle=|d(i, k, l, m ; n)| m-k \geq 0, m-l \geq$ $0 ; i, k, l, m, n=0,1,2, \cdots\}$, i.e.

$$
|d(i, k, l, m ; n)\rangle=\frac{1}{i ! k !}\left(\hat{Q}_{+}^{\dagger} \hat{Q}_{-}^{\dagger}\right)^{i}\left(\hat{P}_{+}^{\dagger}\right)^{k}\left(\hat{P}_{-}^{\dagger}\right)^{l}\left(\hat{O}_{+}^{\dagger}\right)^{m-k}\left(\hat{O}_{-}^{\dagger}\right)^{m-l}\left|D_{n}\right\rangle \quad(i, k, n=0,1,2, \cdots),
$$

which is constructed by acting $\left(\hat{Q}_{+}^{\dagger} \hat{Q}_{-}^{\dagger}\right) i$ times, $\hat{P}_{+}^{\dagger} k$ times, $\hat{P}_{-}^{\dagger} l$ times $\hat{O}_{+}^{\dagger} m-k$ times and $\hat{O}_{-}^{\dagger}$ $m-l$ times on $\left|D_{n}\right\rangle$. Only when $i=k=l=m=0$, the larger degeneracy class reduces to the special dark-state subset $\left\{\left|D_{n}\right\rangle \mid n=0,1,2, \cdots\right\}$ of present five-level atomic system. However, following the method developed in Refs. [8, 9, 10, it is straightforward to confirm that any transition from dark states to other zero-eigenvalue subspace is also forbidden and therefore the robustness of present general EIT quantum memory technique is still perfect, even in the large zero-degeneracy case.

\section{Conclusions and further discussions}

To sum up, the single-ensemble composed of multi-level atoms and multi-ensemble composed of threelevel atoms with EIT are studied in detail in this paper, focused on the interesting dynamical symmetry and its applications to quantum information processing. The general definition of dark-state polaritons (DSPs), and then the dark-states of these different systems are obtained by discovering the symmetrical Lie group of various atomic systems, such as single-atomic-ensemble composed of complex $m$-level $(m>3$, multi-level $)$ atoms, and multi-atomic-ensemble system composed of of three-level atoms. It is interesting that the symmetrical properties of the multi-level system and multi-atomic-ensemble system are dependent on some characteristic parameters of the EIT system. Furthermore, a controllable scheme to generate quantum entanglement between light fields or different atomic ensembles via quantized DSPs theory is discussed, which might be experimentally implemented in the near future..

It is noteworthy that there are many counterparts between the multi-level (single-ensemble) and multi-ensemble atomic systems. For example, the entanglement between two light fields (or among three light fields) can be generated using four- (or five-) level system, and the entanglement between two (or among three) ensembles of atoms can be generated via two- (or three-) atomic-ensemble system; The dynamical symmetry of four-level system is governed by the Lie group $S U(3) \bar{\otimes} H_{3}$, while that of two-atomic-ensemble system is partly governed by $S O(4) \bar{\otimes} H_{4}$; The symmetrical properties of multi-level system is dependent on the parameter of coupling constant $g_{i}$ of probe fields while that of multi-atomic-ensemble system is dependent on the Rabi-frequency of control fields, and the

larger degeneracy class of multi-level system is just similar to that of the corresponding multi-atomicensemble system, etc.. All these interesting aspects may deserve further study in next work.

We thank professors Yong-Shi Wu and J. L. Birman for valuable discussions. This work is supported by NSF of China under grants No.10275036 and No. 10304020, and by Wuhan open fund of state key laboratory of magnetic resonance and atomic and molecular physics, No. T152505.

\section{References}

[1] S. E. Harris, J. E. Field and A. Kasapi, Phys. Rev. A 46, R29 (1992); M. O. Scully and M. S. Zubairy, Quantum Optics (Cambridge University Press, Cambridge 1999). 
[2] L. V. Hau et al., Nature (London) 397, 594 (1999); M. M. Kash et al., Phys.Rev.Lett.82, 529(1999); C. Liu, Z. Dutton, C. H. Behroozi and L. V. Hau, Nature (London) 409, 490 (2001); D. F. Phillips, A. Fleischhauer, A. Mair, R. L. Walsworth and M. D. Lukin, Phys. Rev. Lett. $86,783(2001)$;

[3] M. D. Lukin and A. Imamoğlu, Phys. Rev. Lett. 84, 1419 (2000); M. D. Lukin, S. F. Yelin and M. Fleischhauer, Phys. Rev. Lett. 84, 4232 (2000); M. Fleischhauer and S. Q. Gong, Phys. Rev. Lett. 88, 070404 (2002); C. Mewes and M. Fleischhauer, Phys. Rev. A 66, 033820 (2002).

[4] Y. Wu, J. Saldana and Y. Zhu, Phys. Rev. A 67, 013811 (2003); Y. Li, P. Zhang, P. Zanardi and C. P. Sun, quant-ph/0402177 (2004); G.Juzeliūnas and P.Öhberg, Phys.Rev.Lett. 93, 033602(2004); L. M. Kuang and L. Zhou, Phys. Rev. A 68, 043606 (2003); X. J. Liu, H. Jing and M. L. Ge, Phys. Rev. A 70, 055802 (2004).

[5] M. Fleischhauer and M. D. Lukin, Phys. Rev. Lett. 84, 5094 (2000); M. Fleischhauer and M. D. Lukin, Phys. Rev. A 65, 022314 (2002).

[6] M. D. Lukin, Rev. Mod. Phys. 75, 457 (2003).

[7] Irreversible Quantum Dynamics, Edited by F. Benatti and R. Floreanini, "Decoherece-Free subspace and Subsystem" by D. A. Lidar and B. Whaley, pp. 83-120, Springer Lecture Notes in Physics vol.622, Berlin, (2003); e-print quant-ph/0301032(2003).

[8] C. P. Sun, Y. Li and X. F. Liu, Phys. Rev. Lett. 91, 147903 (2003).

[9] X. J. Liu, H. Jing, X. T. Zhou and M. L. Ge, Phys. Rev. A, 70,015603(2004); X. J. Liu, H. Jing and M. L. Ge, quant-ph/0403171

[10] H. Jing, X. J. Liu, M. L. Ge and M. S. Zhan, Phys. Rev. A 71, 062336 (2005).

[11] R. H. Dick, Phys. Rev. 93, 99 (1954).

[12] B. G. Wybourne, Classical Groups for Physicists (John Wiley, NY, 1974); M. A. Shifman, Particle Physics and Field Theory, p775 (World Scientific, Singapore, 1999).

[13] F. T. Hioe, Phys. Rev. A, 32, 2824 (1985); Phys. Rev. A 28, 879 (1983).

[14] A. B. Matsko, et al., At. Mol. Opt. Phys. 46, 191 (2001); A. S. Zibrov et al., Phys. Rev. Lett. 88, 103601 (2002).

[15] A. André and M. D. Lukin, Phys. Rev. Lett. 89, 143602 (2002)); A. Raczyński and J. Zaremba, Opt. Commun. 209, 149 (2002); quant-ph/0307223 (2003).

[16] M. D. Lukin, S. F. Yelin and M. Fleischhauer, Phys. Rev. Lett., 84, 4232 (2000); E. Arimondo, Progr. In Optics 35, 259 (1996).

[17] J. J. Slosser and G. J. Milburn, Phys. Rev. Lett. 75, 418(1995).

[18] O. Hirota, quant-ph/0101096(2001). 
[19] D. N. Matsukevich and A. Kuzmich, Science, 306, 663 (2004); C. H. van der Wal et al., Science, 301, 196 (2003); O. Mandel et al., Science, 425, 937 (2003); K. Hammerer et al., arXiv: quant-ph/0312156 (2003).

[20] T. Chanelière et al., Nature 438, 833 (2005); M. D. Eisaman et al., Nature 438, 837 (2005).

[21] M. Paternostro, M. S. Kim, and B. S. Ham, Phys. Rev. A 67, 023811 (2003);

[22] Xiaoguang Wang and Barry C. Sanders, Phys. Rev. A 65, 012303 (2003); F. L. Kien et al., Phys. Rev. A 68, 063803 (2003); N. A. Ansari et al., Phys. Rev. A 50, 1492 (1994).

[23] V. Coffman, J. Kundu and W. K. Wootters, Phys. Rev. A 61, 052306 (2000); M. A. Nielsen and I. Chuang, Quantum Computation and Quantum Information (Cambridge University Press, Cambridge, 2000).

[24] C. H. Bennett, D. P. DiVincenzo, J. A. Smolin, and W. K. Wootters, Phys. Rev. A 54, 3824 (1996); R. Laflamme, C. Miquel, J.-P. Paz, and W. H. Zurek, Phys. Rev. Lett. 77, 198 (1996).

[25] A. Dantan, A. Bramati and M. Pinard, Europhys. Lett. 67, 881 (2004); V. Josse, A. Dantan, A. Bramati, M. Pinard and E. Giacobino, Phys. Rev. Lett. 92, 123601 (2004). 